\title{
El comportamiento de la demanda de tabaco en México: 1992-1998
}

\author{
Sergio Sesma-Vázquez, M en Econ Salud, ${ }^{(1)}$ Julio César Campuzano-Rincón, MSP, ${ }^{(1)}$ \\ Víctor Gerardo Carreón-Rodríguez, PhD, (2) Felicia Knaul, PhD, (3) \\ Francisco Javier López-A ntuñano, MD, MPH, ,1) Mauricio Hernández-Avila, PhD.(1)
}

\section{Sesma-Vázquez S, Campuzano-Rincón JC, Carreón-Rodríguez VG, Knaul F, \\ López-Antuñano FJ, Hernández-Avila M. \\ El comportamiento de la demanda de tabaco en México: 1992-1998. \\ Salud Publica Mex 2002;44 supl 1:S82-S92.}

El texto completo en inglés de este artículo está disponible en: http://www.insp.mx/salud/index.html

\section{Resumen}

Objetivo. Describir el comportamiento de la demanda de tabaco en México para cuatro momentos en el tiempo:1992, 1994, 1996 y 1998, y adicio nalmente estimar una función de demanda de cigarrillos. Material y métodos Se realizó un estudio transversal con análisis longitudinal, to mando co mo fuentes de información la Encuesta $\mathrm{N}$ acional de Ingreso y Gasto de los Hogares (EN IGH) y la lista de precios de los cigarrillos de la Procuraduría Federal del Consumidor (Profeco). El gasto, el ingreso y los precios se deflactaron a precios de 1994. Se estratificó a la población en quintiles de ingreso real, y solamente se tomaron en cuenta las áreas rurales y urbanas. Se construyeron indicado res para el consumo diario de cigarrillos y cajetillas y se calcularon los precios por cajetilla. Se calculó la prevalencia ajustada. Mediante análisis de regresión lo gísticos y lineales se estableció la inferencia estadística. Se estimó una función de demanda de cigarrillos mediante una regresión lineal multivariada, para encontrar los determinantes socioeconómicos del consumo de cigarrillos. Resultados La prevalencia ajustada de los hogares que reportaron algún gasto en tabaco disminuyó de 22.4 a $9.9 \%$ entre 1992 y 1998. Los hogares asignaron poco más de $4 \%$ de su ingreso a la adquisición de tabaco. Se observó un gradiente entre nivel de ingreso y gasto en cigarrillos. El quintil I (el más pobre) destinó una mayor proporción de su ingreso que los quintiles superiores. El promedio de cigarrillos consumidos diariamente pasó de 7.5 a 9.8 entre

\author{
Sesma-Vázquez S, Campuzano-Rincón JC, \\ Carreón-Rodríguez VG, Knaul F, \\ López-Antuñano FJ, Hernández-Avila M. \\ Tobacco demand \\ in México: 1992-1998. \\ Salud Publica Mex 2002;44 suppl 1:S82-S92. \\ The English version of this paper \\ is available at: http://www.insp. $\mathrm{mx} / \mathrm{salud} / \mathrm{index} . \mathrm{html}$
}

\begin{abstract}
A bstract
Objective. This paper aims at describing the behavior of tobacco's demand in Mexico across four one-year periods: $1992,1994,1996$, and 1998, as well as to estimate a cigarette demand function. Material and Methods. A crosssectional study with longitudinal analysis was conducted. Information sources were the Encuesta $\mathrm{N}$ acional de Ingreso y Gasto de los Hogares (EN IGH) (N ational Survey of Household Income and Spending) (N HSIS) and the tobacco pack prices reported by the Procuraduría Federal del Consumidor (Profeco) (Federal 0 ffice of Consumer's Protection) (FOCP). Spending, income, and prices were deflated to 1994 prices; the population was stratified into quintiles of real income, by rural and urban areas. Indicators of daily consumption of cigarettes and packs were constructed and prices per pack calculated.Adjusted prevalence figures were estimated. Logistic and linear regression models were used for statistical inference; a cigarette demand function was estimated using multivariate lo gistic regression, to find socioeconomic determinants of cigarette consumption. Results. The adjusted prevalence of household to bacco spending fell from 22.4 to $9.9 \%$ between 1992 and 1998 . Households allocated more than $4 \%$ of their income to tobacco consumption. A trend between income level and cigarette spending was observed, with the first quintile (the poorest population) allocated a greater share of their income than higher quintiles. The average daily consumption of
\end{abstract}

(1) Instituto N acional de Salud Pública, C uernavaca, Morelos, México

(2) Centro de Investigación y Docencia Económicas, México, D.F., México.

(3) Fundación Mexicana para la Salud, México, D.F., México.

Fecha de recibido: 6 de junio de 2001 - Fecha de aprobado: 5 de noviembre de 2001 Solicitud de sobretiros: Sergio SesmaVázquez. Delfín Madrigal N 0.95, edificio B-3, D epto. 704, colonia Santo D omingo, D elegación Coyoacán, 04369 México, D.F.

Correo electrónico: sergio_sesma@ hotmail.com 
1992 y 1998. Se estimó que más de $90 \%$ de los "hogares fumadores" consumió hasta una cajetilla diaria. La demanda estimada de cigarrillos sin filtro aumentó entre 1992 a 1998, pasando de 0.4 a $4.8 \%$, registrándose así el mayor incremento en 1996. Finalmente, se encontró que, tanto los precios co mo el ingreso, fueron los determinantes más importantes del gasto en tabaco. Conclusiones. Una política de precios podría favorecer la reducción de la demanda y, por ende, mejorar las expectativas de salud de la población. El texto completo en inglés de este artículo está disponible en:http:/ /www.insp.mx/salud/index.html

Palabras clave: hogar fumador; consumo de cigarillos y cajetillas; demanda de cigarillos con y sin filtro; gasto en tabaco y política financiera; México cigarettes increased from 7.5 to 9.8 between 1992 and 1998. It was estimated that $90 \%$ of "smoker homes" consumed up to one package per day. The proportion of non-filter cigarettes increased from 0.4 to $4.8 \%$ between 1992-1998, with a bigger increase in 1996. Finally, it was found that the most important determinants of spending were prices and income. Conclusions. Policies focusing on tobacco prices would help to reduce tobacco consumption and improve the health of the Mexican population.

Key words: smoking household; cigarettes and package consumption; filter and tiped-filter demand; expenditure, tobacco financial policy; Mexico a exposición directa e indirecta al humo de tabaco está asociada con un incremento importante en el riesgo de padecer cáncer de pulmón, enfermedad pulmonar obstructiva crónica (EPOC) y enfermedades isquémicas cardiacas. Entre las repercusiones más importantes del consumo de tabaco sobresalen la muerte prematura y el aumento de los costos de atención de los padecimientos asociados con esta adicción.

Diversos autores han estimado el peso de la enfermedad atribuible al tabaquismo, encontrando que en el mundo poco más de 1 millón de muertes anuales son por cáncer de pulmón, equivalentes a $80 \%$ de este tipo de neoplasias. ${ }^{1}$ Asimismo se ha estimado que $45 \%$ de las muertes por enfermedades isquémicas cardiacas, $51 \%$ de las enfermedades cerebrovasculares y $84 \%$ de los enfisemas y las bronquitis crónicas son atribuibles al tabaquismo. ${ }^{2}$ Los costos económicos de la atención a la salud por enfermedades atribuibles al consumo de tabaco han sido calculados por diversos autores en los Estados Unidos de América. La estimación de éstos para enfermedades respiratorias y circulatorias como enfisema pulmonar, infartos, neoplasias, entre otras, varía entre los autores de 8 a 53 billones de dólares anuales. ${ }^{3-5}$ Recientemente, la industria tabacalera fue penalizada con 145 mil millones de dólares por daños a la salud en el estado de Florida. ${ }^{6}$ Esta última cifra indica que la contribución de las empresas tabacaleras se encuentra muy por debajo del peso económico de la enfermedad que provocan sus productos.

El consumo de cigarrillos en el área de las Américas entre 1990 y 1995 muestra una tendencia decreciente en la región norte del continente donde se encuentran los Estados Unidos y Canadá, mientras que en las regiones del Centro y Sudamérica, por el contrario, se observa un ligero crecimiento en la demanda, sin llegar a los niveles de consumo de los países antes mencionados. ${ }^{7}$ Lo anterior podría explicarse por la penetración de las transnacionales tabacaleras a estos mercados, como consecuencia de las restricciones impuestas en los países de altos ingresos.

En la Encuesta Nacional de Salud (ENSA) de 1986 se encontró que la prevalencia de fumadores fue de $17.5 \%$. Asimismo se estimó que dentro de la población fumadora, la proporción de individuos que fumaron entre 1 a 15 cigarrillos diarios fluctuó entre 81.6 y $84.5 \% .8,9$ Datos de la Encuesta Nacional de Adicciones (ENA) indican que la prevalencia entre 1988 y 1998 pasó de 25.8 a $27.7 \%{ }^{10}$

La demanda de tabaco en México se concentra principalmente en los cigarrillos, los cuales se dividen en cigarrillos con y sin filtro, cuya característica esencial es que los primeros son más caros que los segundos.

La evolución del consumo de tabaco puede explicarse mediante el análisis del comportamiento de la producción y el gasto agregado de los hogares. Entre 1994 y 1996, la producción de cigarrillos con y sin filtro creció 4.5 y $44.3 \%$, respectivamente. En cambio, para el bienio 1996-1998, dicha producción aumentó en los primeros (14.1\%) mientras que decreció para los cigarrillos sin filtro (26.5\%). ${ }^{11}$ De acuerdo con las Cuentas Nacionales, el consumo de tabaco de origen nacional representó 98\% del gasto de los hogares para el periodo mencionado, lo que significa que el intercambio comercial de tabaco con el exterior es relativamente pequeño. $^{12}$

Con la información anterior se puede estimar que, en 1994, para la población de 12 a 65 años, la producción per capita correspondía a 41 cajetillas, mientras que en 
1998 equivalía a poco más de 50. Adicionalmente, bajo el supuesto de que cada hogar tuviera cuatro miembros en promedio y contando con que una cajetilla contiene 20 cigarrillos, estimamos que la producción diaria de cigarrillos por hogar pasó de 9 a 11 cigarrillos entre 1994 y 1998. Lo anterior puede interpretarse como que el consumo de cigarrillos en México ha aumentado y que los fumadores han trasladado su demanda a cigarrillos sin filtro de más bajo precio desde 1994, año en que se presentó una severa crisis económica nacional.

La evidencia respecto de los determinantes socioeconómicos que regulan el comportamiento de la demanda de tabaco señalan al precio y al ingreso como algunos de los factores determinantes de mayor impacto sobre el consumo de tabaco. Asimismo, aspectos como la edad, la educación, el tamaño de familia, el género, la venta ilegal (contrabando), entre otras, han sido incorporadas como variables de control dentro de los modelos sugeridos. Los resultados sostienen, principalmente, que los precios y el consumo tienen una relación inversa, es decir que cuando los primeros se incrementan, el consumo disminuye. ${ }^{13-18}$

La edad ha sido considerada como un elemento importante, particularmente dentro de los modelos económicos de la adicción, en donde se menciona que el consumo actual de los fumadores adultos está condicionado por el consumo en la adolescencia. ${ }^{19}$ Esto se refleja en una estimación de la ENA de 1998, en donde se calcula que el promedio de cajetillas consumidas al año, en una población adolescente de entre 12 y 14 años, fue de 26.7 mientras que en una población de 55 a 65 años el consumo promedio fue 133.8 cajetillas. Asimismo, el consumo por género indica que los hombres fuman 115 cajetillas, en promedio al año, mientras que las mujeres consumen 85. Conviene añadir que los hombres redujeron su consumo $26 \%$ y las mujeres $19 \%$, de acuerdo con las ENA, entre 1988 y $1998 .{ }^{10} \mathrm{La}$ relación entre el tamaño del hogar y el consumo no ha sido estudiada pero es una variable que determina la demanda de tabaco al interior de cada hogar. El signo esperado en la educación podría ser ambiguo ya que a mayor educación mayor información sobre el efecto negativo del tabaquismo y, por ende, menor consumo; pero, por otro lado, a mayor escolaridad, mejor puesto de trabajo, más responsabilidad laboral y mayor estrés que induce a las personas a demandar tabaco. También existen otros factores no económicos que determinan la demanda: la publicidad, factores culturales, el grado de adicción, las actitudes de los jóvenes hacia el riesgo, la delimitación de áreas exclusivas para fumadores y la venta de cigarrillos a menores. ${ }^{19-24}$

La determinación de los factores socioeconómicos asociados con la demanda resulta fundamental en la elaboración de planes y políticas contra el tabaquismo. Particularmente, el análisis de los determinantes económicos permitiría la utilización de la teoría económica como una herramienta vital dentro del ámbito de la salud pública, para controlar el consumo de tabaco. La contención en el consumo de tabaco repercutiría positivamente en la salud de la población fumadora y no fumadora, además generaría un ahorro importante de recursos financieros al sector salud.

\section{Material y métodos}

Los datos para este estudio se obtuvieron a partir de la Encuesta Nacional de Ingreso y Gasto de los Hogares (ENIGH) de 1992, 1994, 1996 y 1998. La línea basal para estimar el consumo de cigarrillos con y sin filtro se definió a partir de información proporcionada por la Procuraduría Federal del Consumidor (Profeco) en materia de precios de cigarrillos por marca. El gasto mensual o diario se consideró en términos reales a pesos de 1994. Se estratificó a la población en quintiles de ingreso trimestral real. En este estudio las áreas urbanas están definidas como poblaciones de más de 100 mil habitantes y las zonas metropolitanas, y las áreas rurales como poblaciones de menos de 2500 habitantes.

La ENIGH, realizada por el Instituto Nacional de Estadística Geografía e Informática (INEGI) cada dos años, es un instrumento que recoge información sobre la distribución, monto y estructura del ingreso y el gasto de los hogares con el propósito de evaluar los cambios en el nivel de vida de la población. ${ }^{25}$ El gasto en tabaco se divide en tres categorías: cigarrillos, puros y tabaco picado. Las fechas del levantamiento de las ENIGH fue siempre durante el tercer trimestre del año.

La información se recolecta por medio de un cuadernillo que contiene preguntas relacionadas con características socioeconómicas y ocupacionales de los miembros del hogar, así como de las transacciones económicas, entendidas éstas como ingreso-gasto. El cuadernillo se llena por medio de entrevistas directas, y se completa con los datos proporcionados por el informante quien mantiene el instrumento en su poder durante siete días, supervisado constantemente por el encuestador. Las preguntas relacionadas con el consumo de tabaco han variado entre encuestas. En la ENIGH de 1992, la pregunta era específica: ¿qué cantidad de cigarrillos, puros y tabaco picado compró en el transcurso de la semana? ${ }^{25}$ Mientras que en las ENIGH de 1994 a 1998 la instrucción era: Anote los alimentos, bebidas alcohólicas y no alcohólicas, y tabaco que compró usted y los alimentos del hogar el día de ayer. Si algún miembro del hogar consumió fuera de la casa el desayuno, la comida, la cena o alguna entre comi- 
da en algún restaurante, lonchería, cafetería, etcétera, anote este consumo. ${ }^{26}$ Esta situación podría generar, igualmente, ciertos problemas de comparabilidad entre las encuestas.

La unidad de observación fueron los hogares y sus miembros y, a su vez, se seleccionaron los hogares que se encuestaron a partir de un marco muestral maestro. La muestra fue de más de 10 mil hogares clasificados por zonas de residencia rurales y urbanas, distribuidas por toda la República mexicana. El diseño de la muestra fue probabilístico, polietápico, estratificado y por conglomerado. El tamaño de la muestra utilizado para los años de este estudio fue 10530 en 1992; 12815 en 1994; 14042 en 1996 y 10601 en 1998. Se tomaron como criterios de exclusión en este estudio: a) los hogares que no reportaron ingreso, y b) los hogares con ausencia del jefe del hogar.

Para este estudio se consideró como "hogar fumador" a todo aquel que respondió haber realizado un gasto positivo en tabaco durante la semana en la que se realizó la encuesta. Asimismo, en la ENIGH se recolectó información sobre el consumo y los precios de tabaco en kilogramos, y de manera semanal. A partir de esta información se hicieron varias transformaciones para estimar el consumo en cigarrillos y cajetillas. El primer paso consistió en suponer que 800 cigarrillos corresponden a $1 \mathrm{Kg}$. de tabaco y, por ende, a 40 cajetillas de 20 cigarrillos cada una. El total de cajetillas consumidas diariamente (CCD) se obtuvo a partir de la siguiente fórmula:

\section{Cajetillas consumidas diariamente \\ $(C C D)=\left[\left(\right.\right.$ cantidad en $\left.\left.\mathrm{Kg}^{*} 40 / 7\right)\right]$}

El consumo semanal y mensual de cajetillas se calculó multiplicando CCD por 7 y 30 , respectivamente. El consumo diario de cigarrillos se obtuvo multiplicando CCD por 20. Los precios por cajetilla (PPC) se obtuvieron a través del gasto real en tabaco reportado por la encuesta y se calcularon por medio de la siguiente fórmula.

Precio por cajetilla $(\mathrm{PPC})=\left[\right.$ Gasto semanal real $\left./\left(\mathrm{CCD}^{*} 7\right)\right]$

En la figura 1 presentamos la comparación de los precios promedio de los cigarrillos reportados por la Profeco, los estimados en Encuesta Industrial Anual y los precios por cajetilla (PPC).

A partir de los PPC se estimó el consumo de cigarillos con y sin filtro, tomando como precio basal el precio real reportado por la Profeco de los cigarrillos sin filtro más demandados en el mercado, "Delicados", de acuerdo con un estudio en el estado de Morelos no publicado. ${ }^{10}$ De esta forma, el consumo de cajetillas con filtro (CCF) y sin filtro (CSF) se calculó de la siguiente manera:

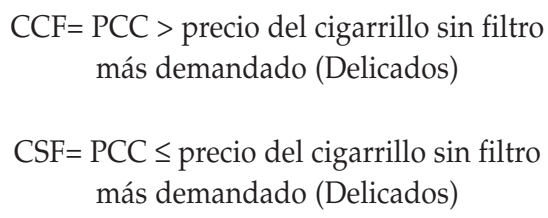

Se hicieron análisis de regresión, tanto lineales como logísticos, para observar la significancia estadística de los cambios en la tendencia del consumo de cigarrillos y cajetillas, en la demanda de cigarrillos sin filtro y en las proporciones de los hogares que efectuaron algún gasto en tabaco.

Se calcularon la prevalencia ajustada de "hogares fumadores" y la proporción ajustada en el "consumo de cajetillas" $\mathrm{y}$ "consumo de cigarrillos sin filtro" dentro de "hogares fumadores". Tanto la prevalencia como la proporción ajustada se estimaron a partir de un modelo Logit:

$X=\beta_{0}+\beta_{1}{ }^{*}$ ENIGH $+\beta_{2}{ }^{*}$ Quintil $+\beta_{3}{ }^{*}$ Género $+\beta_{4}{ }^{*}$ Tam $\_$hog $+\beta_{5}{ }^{*}$ Educ $+U_{t}$

Donde:

- X es "hogares con gasto en tabaco". $X=1$, si el hogar consume tabaco y $X=0$, si el hogar no consume tabaco para el caso de la prevalencia. Para el caso de las proporciones ajustadas, $\mathrm{X}$ es el consumo de cajetillas $\mathrm{u}$ hogares con consumo de cigarrillos sin filtro. $X=0$, si el hogar no consume tabaco y $X=1$, si el hogar consume tabaco.

- ENIGH es una variable indicadora para el año de levantamiento de la información: para los años 1992, 1994, 1996 y 1998;

- Quintil es una variable categórica que indica el estrato de ingreso de cada hogar.

- Género es el sexo del jefe del hogar. 1 si es hombre y 0 si es mujer.

- Tam_hog es una variable dummy que indica el número de dependientes de cada hogar (tamaño del hogar). 1 si es igual o menor a 4 y 0 superior a cuatro miembros. El valor de 4 señala el promedio de individuos de cada hogar.

- Educ es una variable categórica que señala la escolaridad del jefe del hogar.

- $U_{t}$ es el término de error generado por variables no contempladas en el modelo.

La prevalencia ajustada y la proporción ajustada se calcularon con la siguiente fórmula:

$$
\mathrm{P}_{\text {AJUSTADA }}=\mathrm{e}^{\mathrm{bi}} /\left[\left(1+\mathrm{e}^{\mathrm{bi}+\mathrm{bi}+1}\right)\right]
$$


1.1. Precios estimados de los cigarrillos sin filtro, México

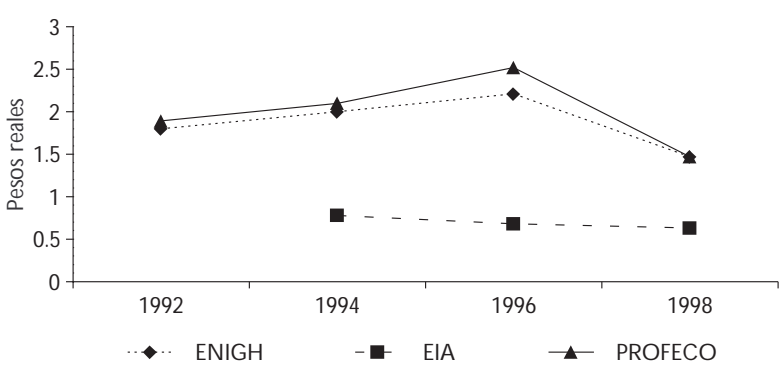

Los precios de la Profeco son promedio de los cigarrillos sin filtro

Los precios de los cigarrillos sin filtro cayeron entre 1996 y 1998, lo que favoreció el consumo

1.3. Prevalencia ajustada del consumo de cigarrillo sin filtro y producción de cajetillas sin filtro de acuerdo con la Encuesta Industrial A nual (EIA), México

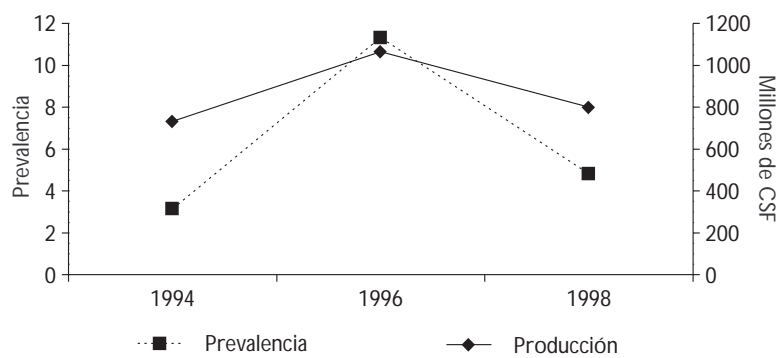

La estimación de la demanda de cigarrillos sin filtro coincide con la producción de cajetillas de este tipo de cigarrillos. Aumentó entre 1994 y 1996 a consecuencia de la crisis económica y se redujo en 1998 por la disminución de los precios y la recuperación de la capacidad adquisitiva de los fumadores

EN IGH: Encuesta $\mathrm{N}$ acional de Ingreso y $\mathrm{G}$ asto de los Hogares

EIA: Encuesta Industrial A nual

Profeco: Procuraduría Federal del Consumidor

CSF: Cajetillas sin filtro

IEPS: Impuestos Especiales a la Producción y los Servicios
1.2. Precios estimados de los cigarrillos con filtro, México

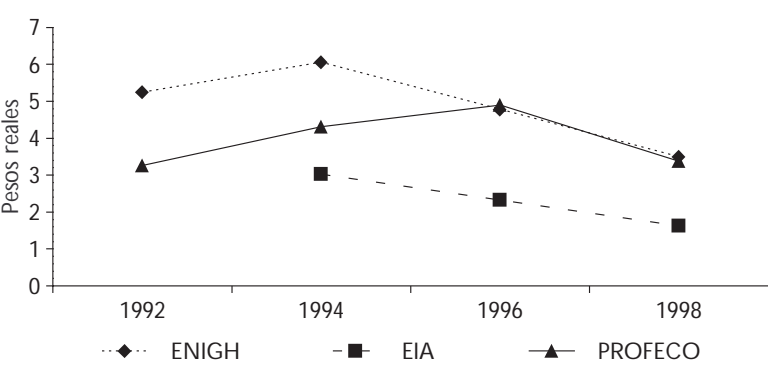

Los precios de la Profeco son promedio de los cigarrillos sin filtro

Los precios de los cigarrillos con filtro cayeron entre 1996 y 1998, lo que favoreció el consumo

1.4. Impuestos Especiales a laPproducción y los Servicios (IEPS) de los cigarrillos con filtro y la prevalencia ajustada de "hogares fumadores", México

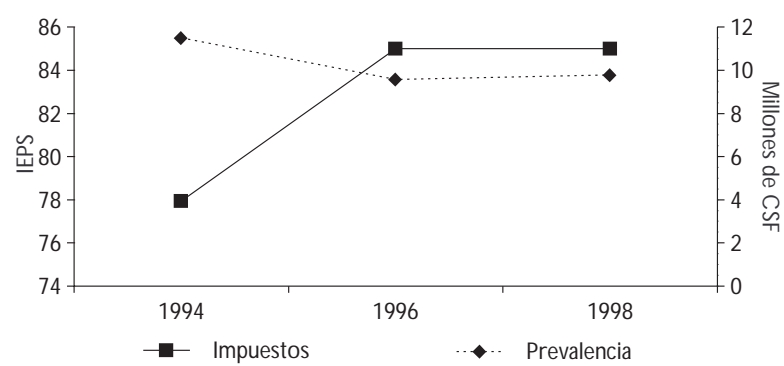

Uno de los argumentos a favor de los impuestos es que puede reducir la prevalencia, lo que favorece las expectativas de salud de la población fumadora y no fumadora

Figura 1. Comparación de precios promedio de cigarrillos, según la Procuraduría Federal del Consumidor, la Encuesta Industrial Anual y la Encuesta Nacional de Ingreso y Gasto de los Hogares

Donde:

- $\mathrm{P}_{\text {Ajustada }}$ es la prevalencia ajustada o la proporción ajustada

- e $e^{b i}$ es la exponencial del coeficiente de la regresión logit (b) de la ENIGH en 1992.

- $\mathrm{e}^{\mathrm{bi+1}}$ es la exponencial del coeficiente de la regresión logit (b) de las ENIGH posteriores.

Asimismo, se estimaron diversas regresiones lineales multivariadas para encontrar algunos de los determinantes socioeconómicos de la demanda de tabaco en México, condicionados por año de la encuesta, quintil de ingreso y lugar de residencia. El modelo estimado fue el siguiente:

$$
\begin{gathered}
\text { Cons }_{\mathrm{t}}=\beta_{0}+\beta_{1}{ }^{*} \text { Prec }_{\mathrm{t}}+\beta_{2}{ }^{*} \text { Ingre }_{\mathrm{t}}+\beta_{3}{ }^{*} \text { Género }_{\mathrm{t}}+\beta_{4}{ }^{*} \text { Edad }_{\mathrm{t}}+\beta_{5} \\
{ }^{*} \text { Educ }_{\mathrm{t}}+\beta_{6}{ }^{*} \text { Tam }_{-} \text {hog }_{\mathrm{t}}+\mathrm{U}_{\mathrm{t}}
\end{gathered}
$$

Donde:

- Cons ${ }_{t}$ es el consumo semanal de cajetillas en el año t.

- $\beta_{0}$ es el consumo de tabaco independiente de cualquier otra variable.

salud pública de méxico / vol.44, suplemento 1 de 2002 
- Prec $_{t}$ es el precio de la cajetilla consumida en el año $t$.

- Ingre ${ }_{t}$ es el ingreso semanal monetario del hogar en el año t.

- Género ${ }_{t}$ corresponde al sexo jefe del hogar en el año t.

- Edad $_{t}$ es la edad del jefe del hogar en el año t.

- Educ $c_{t}$ corresponde al grado de estudios de estudio del jefe del hogar en el año t;

- Tam_Hog es el número de residentes en el hogar encuestado en el año t.

- $U_{t}$ es el término de error generado por variables no contempladas en el modelo.

Se categorizaron las variables edad, educación y tamaño del hogar. Para la edad del jefe se establecieron tres categorías: a) 15-35 años, b) 36-50 años y c) 51 y más años. La educación se clasificó en: 1) sin instrucción, 2) hasta secundaria, 3) hasta estudios superiores y 4) posgrado. El tamaño del hogar se categorizó en: hasta cuatro miembros y más de cuatro, que es el promedio de residentes en el hogar.

Finalmente, se realizaron las pruebas típicas de detección de anomalías en una regresión lineal como heteroscedasticidad y multicolinealidad. En la primera se aplicó el test de Goldfeld-Quandt mientras que para la segunda se hizo la prueba de Factores de Inflación de la Varianza (VIF), utilizando el paquete estadístico STATA 7.0.

\section{Resultados}

Los resultados se presentan en el siguiente orden: la evolución que han tenido el gasto y el consumo, tanto por nivel de ingreso como por lugar de residencia, de acuerdo con la ENIGH. Finalmente, presentaremos algunos de los determinantes de la demanda de cigarrillos en nuestro país para el periodo de análisis.

\section{Nivel de análisis I: Gasto en tabaco}

La descripción horizontal del cuadro I muestra que la prevalencia ajustada promedio de los hogares que reportaron gasto en tabaco disminuyó de 21.8 a 9.6\% entre 1992 y 1996, pero se incrementó ligeramente en 1998 a 9.9\%. La descripción vertical del cuadro I muestra la relación directa entre estrato socioeconómico y hogares con gasto en cigarrillos. Los resultados indican que cuando la capacidad de compra aumenta, entonces resulta más frecuente encontrar "hogares fumadores". Mediante un análisis de regresión logística se determinó que las variaciones observadas fueron estadísticamente significativas entre 1992 y 1998, controlando por nivel de ingreso, tamaño del hogar, lugar

\begin{tabular}{|c|c|c|c|c|}
\hline \multicolumn{5}{|c|}{$\begin{array}{l}\text { Prevalencia AJUSTADA* DE hOGARES CON GASTO } \\
\text { Mensual en tABACO: 1992-1998, MÉxico }\end{array}$} \\
\hline Estrato & 1992 & 1994 & 1996 & 1998 \\
\hline Promedio ${ }^{\ddagger}$ & 21.8 & $11.6^{\S}$ & $9.6^{\S}$ & $9.9^{\S}$ \\
\hline Urbano" & 25.8 & $11.8^{\S}$ & $12.2^{\S}$ & $10.0^{\S}$ \\
\hline Rural\# & 19.7 & $11.1^{\S}$ & $9.4^{\S}$ & $9.5^{\S}$ \\
\hline Q uintil I\# & 15.2 & $7.9^{\S}$ & $6.9^{\S}$ & $5.8^{\S}$ \\
\hline Q uintil II & 17.1 & $9.5^{\S}$ & $8.2^{\S}$ & $9.0^{\S}$ \\
\hline Q uintil III\# & 21.5 & $12.0^{\S}$ & $8.7^{\S}$ & $9.6^{\S}$ \\
\hline Q uintil IV\# & 23.9 & $12.4^{\S}$ & $10.3^{\S}$ & $12.2^{\S}$ \\
\hline Q uintilV\# & 28.2 & $15.0^{5}$ & $12.3^{\S}$ & $11.5^{\S}$ \\
\hline \multicolumn{5}{|c|}{$\begin{array}{l}\text { * Ajustado por nivel de ingreso, tamaño del hogar, educación, edad y gé } \\
\text { nero del jefe del hogar y zona de residencia } \\
\text { ₹ Incluye todos los estratos de ingreso y zonas de residencia } \\
\text { \& Cambios estadísticamente significativos con un } \alpha=0.05 \text { y } 1992 \text { como } \\
\text { año de referencia } \\
\text { \# Incluye toda la población fumadora y no fumadora }\end{array}$} \\
\hline
\end{tabular}

\section{Cuadro II \\ Gasto mensual* destinado a CigarRiLlos: 1992-1998, MÉXICO}

\begin{tabular}{|c|c|c|c|c|c|c|c|c|}
\hline \multirow[t]{2}{*}{ Estrato } & \multicolumn{2}{|c|}{1992} & \multicolumn{2}{|c|}{1994} & \multicolumn{2}{|c|}{1996} & \multicolumn{2}{|c|}{1998} \\
\hline & 67 & $\$$ & $\%$ & $\$$ & $\%$ & $\$$ & $\%$ & $\$$ \\
\hline romedio\# & 4.7 & 48.8 & $4.2^{\&}$ & 52.0 & $4.3^{\neq}$ & 35.7 & $4.0^{\alpha}$ & 36.3 \\
\hline Jrbano $^{\infty}$ & 2.8 & 55.4 & $2.5^{\S}$ & 57.8 & $3.2^{\&}$ & 42.5 & $2.8^{\alpha}$ & 39.9 \\
\hline Rural & 6.1 & 42.3 & $5.8^{\S}$ & 44.6 & $6.0^{\neq}$ & 27.0 & $6.0^{\alpha}$ & 33.3 \\
\hline Q uintil $\left.\right|^{\infty}$ & 11.5 & 26.2 & $10.7^{\S}$ & 23.5 & $8.9^{\alpha}$ & 17.9 & $10.8^{\&}$ & 21.1 \\
\hline Q uintil $\|^{\infty}$ & 5.9 & 35.1 & $6.4^{\delta}$ & 37.1 & $3.9^{\neq}$ & 22.9 & $4.9^{\neq}$ & 28.4 \\
\hline$\overline{Q \text { uintil }\|\|^{\infty}}$ & 3.6 & 43.8 & $4.6^{\S}$ & 42.8 & $3.8^{\neq}$ & 35.4 & $3.5^{*}$ & 32.6 \\
\hline Q uintil IV ${ }^{\infty}$ & 2.9 & 49.5 & $3.1^{\AA}$ & 46.7 & $2.9^{\neq}$ & 43.5 & $2.7^{\neq}$ & 39.9 \\
\hline Q uintil $V^{\infty}$ & 1.8 & 65.5 & $2.0^{\neq}$ & 77.6 & $1.9^{\&}$ & 63.3 & $1.5^{\S}$ & 50.3 \\
\hline \multicolumn{9}{|c|}{$\begin{array}{l}\text { * Ajustado por nivel de ingreso, tamaño del hogar, educación, edad y gé- } \\
\text { nero del jefe del hogar y zona de residencia } \\
\text { ₹ Gasto como proporción del ingreso real \% } \\
\text { \& Gasto monetario real } \\
\text { \# Incluye todos los estratos de ingreso y zonas de residencia. } \\
\text { \& Cambios estadísticamente no significativos } \\
\text { * Cambios estadísticamente significativos con un } \alpha=0.05 \text { y } 1992 \text { como } \\
\text { año de referencia } \\
\text { ¿ Incluye solamente población fumadora }\end{array}$} \\
\hline
\end{tabular}

de residencia, género, edad y educación del jefe del hogar.

El gasto del hogar destinado al consumo de tabaco aparece en el cuadro II clasificado en gasto monetario y como porcentaje del ingreso. Los estratos 
socioeconómicos altos son los que más gastan en tabaco. Sin embargo, como proporción del ingreso, se puede observar un gradiente decreciente; esto quiere decir que, la asignación presupuestal familiar al gasto en tabaco en los niveles socioeconómicos bajos, es mayor que en los altos, por lo que el gasto destinado al financiamiento de la adicción puede ser considerado como regresivo. En promedio, los hogares destinaron poco menos de $5 \%$ de su ingreso mensual a la adquisición de cigarrillos durante el periodo analizado. En promedio, entre 1992 y 1998, el nivel socioeconómico más bajo (quintil I) destinó más de 10\% de su ingreso al consumo de cigarrillos mientras que el quintil V asignó poco menos de $2 \%$; esto equivale a un desembolso promedio mensual de 20 y 60 pesos, respectivamente. El análisis de regresión lineal permitió determinar que el cambio observado en el promedio del porcentaje del ingreso destinado al consumo de tabaco entre 1992 y 1998, no resultó estadísticamente significativo, controlado por nivel de ingreso, lugar de residencia, tamaño del hogar, género, educación y edad del jefe del hogar.

\section{Nivel de análisis II: estimación del consumo diario de cigarrillos}

Entre 1992 y 1998, el consumo promedio de cigarrillos aumentó poco más de dos cigarrillos diarios. En el cuadro III se observa, verticalmente, un gradiente entre el nivel de ingreso y el consumo; esto es que conforme aumenta la capacidad adquisitiva de la po- blación fumadora, se incrementa la demanda de cigarrillos. Horizontalmente, se muestra que el consumo promedio en los estratos de ingreso más bajo (quintiles I y II) aumentó casi el doble mientras que en los quintiles IV y V, el consumo permaneció casi sin cambio. Adicionalmente, para 1998, el consumo de los quintiles I y V fue básicamente el mismo.

La interpretación por la zona de residencia indica que los hogares urbanos consumen más que los rurales, excepto para 1996, y que la demanda en estas áreas aumentó entre 1992 y 1998. El análisis de regresión lineal permitió observar que los incrementos en el consumo promedio estimado de cigarrillos entre 1992 y 1998 fueron estadísticamente significativos, controlados por precios, nivel de ingreso, lugar de residencia, género, educación y edad del jefe del hogar y por el tamaño del hogar. Sin embargo, en los quintiles I y III, así como en las áreas rurales, las variaciones observadas no fueron significativas.

\section{El consumo diario estimado de cajetillas}

Como se observa de manera horizontal en el cuadro III, la proporción ajustada de "hogares fumadores" mostró que más de $90 \%$ de éstos consumió de 1 a 20 cigarrillos diarios, en promedio. Sin embargo, esta cifra ha ido disminuyendo con el transcurrir de los años. Efectivamente, de 1992 a 1998, esta proporción cayó siete puntos porcentuales, pasando de 95.4 a $88.6 \%$, respectivamente, lo que significa que se han reportado más hogares con consumo superior a una cajetilla

\section{Cuadro III \\ Proporción estimada* de "hogares fumadores" que consumieron hasta unA CAJETILLA DiARIA: 1992-1998, MÉxICO}

\begin{tabular}{|c|c|c|c|c|c|c|c|c|}
\hline \multirow[b]{2}{*}{ Estrato } & \multicolumn{2}{|c|}{1992} & \multicolumn{2}{|c|}{1994} & \multicolumn{2}{|c|}{1996} & \multicolumn{2}{|c|}{1998} \\
\hline & Cigarrillos & Proporción & Cigarrillos & Proporción & Cigarrillos & Proporción & Cigarrillos & Proporción \\
\hline Promedio & 7.5 & 95.4 & $8.0^{\S}$ & $94.6^{\S}$ & $8.8^{\#}$ & $89.3^{\#}$ & $9.8^{\#}$ & $88.6^{\#}$ \\
\hline Urbano\& & 7.6 & 94.9 & $8.5^{\S}$ & $95.3^{\S}$ & $7.8^{\S}$ & $88.2^{\#}$ & $9.6 \#$ & $87.9^{\#}$ \\
\hline Rural\& & 7.6 & 95.0 & $7.7^{\S}$ & $94.7^{\S}$ & $8.7^{\S}$ & $89.8^{\#}$ & $9.1^{\S}$ & $91.3^{\#}$ \\
\hline$\left.\overline{Q \text { uintil }}\right|^{\&}$ & 5.7 & 96.9 & $6.5^{\S}$ & $98.9^{\S}$ & $5.5^{\S}$ & $97.3^{\S}$ & $9.7^{\S}$ & $98.4^{\S}$ \\
\hline Q uintil $\|^{\&}$ & 6.8 & 96.1 & $7.0^{\S}$ & $97.1^{\S}$ & $7.0^{\S}$ & $93.1^{\S}$ & $13.0^{\#}$ & $91.5^{\S}$ \\
\hline$\overline{Q \text { uintil }\|\|^{\&}}$ & 7.6 & 97.0 & $6.8^{\S}$ & $96.6^{\S}$ & $7.6^{\S}$ & $93.2^{\#}$ & $10.8^{5}$ & 92.3\# \\
\hline Q uintil IV\& & 7.3 & 96.8 & $8.0^{\S}$ & $94.0^{\S}$ & $9.2^{\#}$ & $87.9^{\#}$ & $9.5^{\#}$ & $86.2^{\#}$ \\
\hline Q uintil V\& & 8.7 & 92.3 & $9.8^{\S}$ & $90.9^{\S}$ & $15.4^{\#}$ & $82.4^{\#}$ & $9.8^{\#}$ & $84.6^{\#}$ \\
\hline
\end{tabular}

* Ajustado por nivel de ingreso, tamaño del hogar, educación, edad y género del jefe del hogar y zona de residencia

* Incluye todos los estratos de ingreso y zonas de residencia

$\S$ Cambios no estadísticamente significativos

\# Cambios estadísticamente significativos con un $\alpha=0.05$ y 1992 como año de referencia

\& Incluye solamente población fumadora 
diaria. Verticalmente, se muestra un gradiente entre nivel de ingreso y consumo de cajetillas; esto significa que conforme aumenta la capacidad adquisitiva, se incrementa la proporción de hogares que se estima consumen más de una cajetilla diaria.

Por medio de un análisis de regresión logística se observó que la disminución en la proporción ajustada estimada promedio de los "hogares fumadores" entre 1992 y 1998 fue estadísticamente significativa, excepto en 1994. Cabe destacar que las variaciones experimentadas de 1994 a 1998 por los quintiles I y II, tomando como año de referencia 1992, no fueron estadísticamente significativos (hacia la baja), controlado por nivel de ingreso, lugar de residencia, tamaño del hogar, género, educación y edad del jefe del hogar.

\section{La demanda estimada de cigarrillos con y sin filtro}

El consumo de cajetillas con y sin filtro es un dato interesante que se aprecia en el cuadro IV. La prevalencia ajustada estimada de hogares que adquirieron cigarrillos sin filtro aumentó entre 1992 y 1996. Para el primer año, el consumo de este tipo de cigarrillos alcanzó $0.4 \%$ de los hogares mientras que para 1996 aumentó en 11 puntos porcentuales, estableciéndose este indicador en 11.4\%. Sin embargo, para 1998, la demanda de cigarrillos sin filtro decreció casi siete puntos por- centuales con respecto al bienio anterior, lo que puede ser atribuible a una mejora en la capacidad de compra de los hogares fumadores.

En el cuadro IV, se observa la prevalencia estimada de hogares demandantes de cigarrillo sin filtro por estrato socioeconómico. En las áreas rurales, la prevalencia fue mayor que en las urbanas, excepto en 1992. Por nivel de ingreso, se aprecia un gradiente entre ca-

\section{Cuadro IV}

Proporción estimada de hogares Que consumieron CIGARRILLO SIN FILTRO: 1992-1998, MÉXICO

\begin{tabular}{|c|c|c|c|c|}
\hline Estrato & 1992 & 1994 & 1996 & 1998 \\
\hline Promedio* & 0.4 & $3.2^{\ddagger}$ & $11.4^{\ddagger}$ & $4.8^{\ddagger}$ \\
\hline Urbano§ & 0.5 & $1.3^{\ddagger}$ & $7.0^{\ddagger}$ & $4.7^{\ddagger}$ \\
\hline$\overline{\text { Rurals }}$ & 0.4 & $3.9^{\ddagger}$ & $16.8^{\ddagger}$ & $5.3^{\ddagger}$ \\
\hline$\left.\overline{Q \text { uintil I}}\right|^{\S}$ & 0.4 & $4.2^{\ddagger}$ & $22.0^{\ddagger}$ & $6.5^{\ddagger}$ \\
\hline Q uintil $\|^{\S}$ & 0.3 & $4.1^{\ddagger}$ & $19.1^{\ddagger}$ & $8.9^{\ddagger}$ \\
\hline$\overline{Q \text { uintil III }}$ & 0.5 & $3.7^{\ddagger}$ & $8.5^{\ddagger}$ & $2.6^{\ddagger}$ \\
\hline$\overline{Q \text { uintil IV§ }}$ & 0.6 & $4.0^{\ddagger}$ & $7.2^{\ddagger}$ & $4.0^{\ddagger}$ \\
\hline$\overline{Q \text { uintil V§ }}$ & 0.1 & $1.3^{\ddagger}$ & $6.0^{\ddagger}$ & $5.1^{\ddagger}$ \\
\hline \multicolumn{5}{|c|}{$\begin{array}{l}\text { * Incluye todos los estratos de ingreso y zonas de residencia } \\
\text { ₹ Cambios estadísticamente significativos con un } \alpha=0.05 \text { y } 1992 \text { como } \\
\text { año de referencia } \\
\S \text { Incluye solamente población fumadora }\end{array}$} \\
\hline
\end{tabular}

\section{Cuadro V}

Resultados del modelo estadístico de la demanda de Cajetillas. México, 1992-1998*

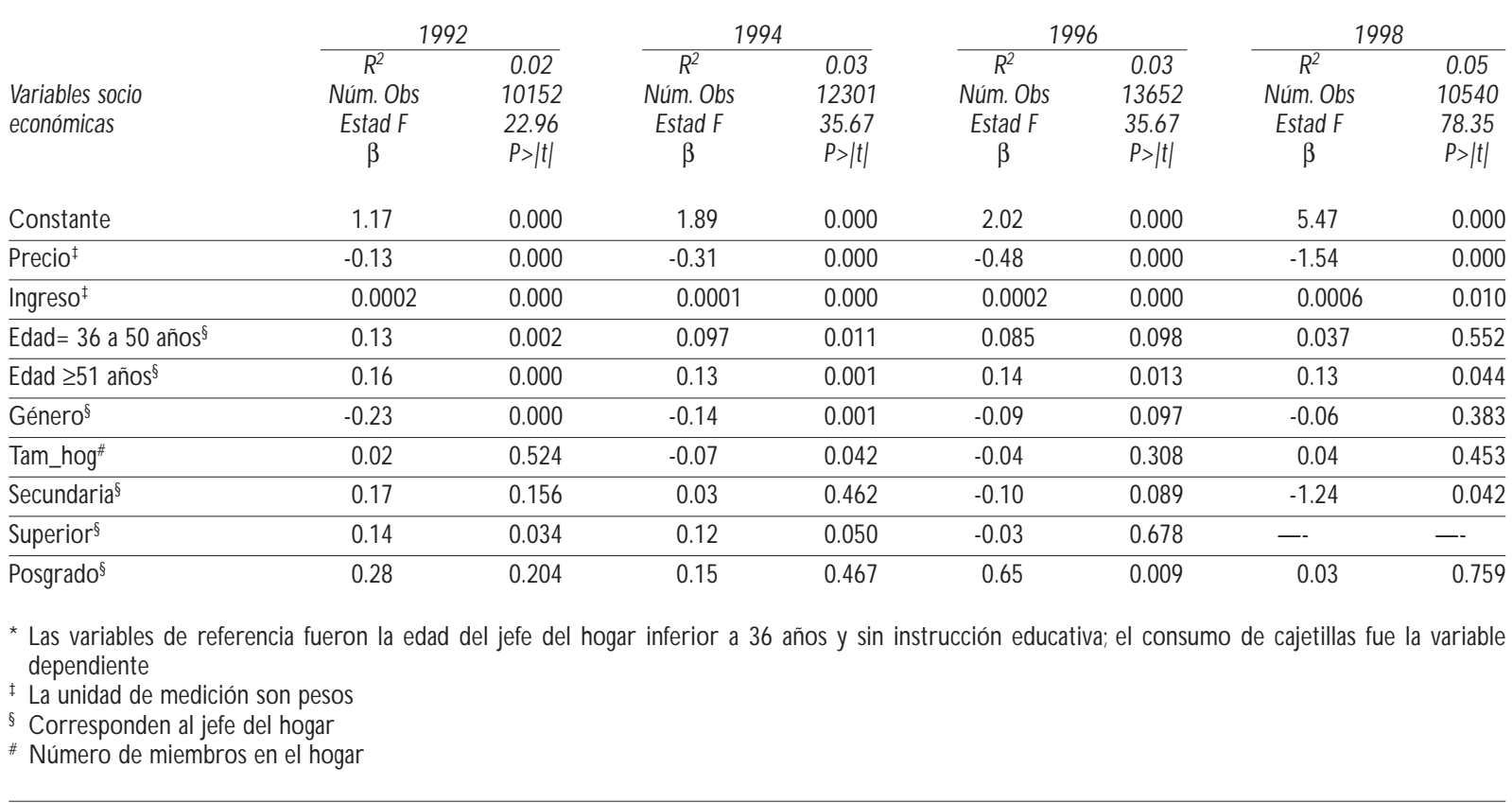


pacidad adquisitiva y la prevalencia; esto es que conforme aumenta el ingreso, la proporción estimada de hogares que consumen tabaco sin filtro disminuye. Por medio de un análisis de regresión logística, se observó que los aumentos promedio en la proporción de hogares que consumieron cigarrillo sin filtro fueron estadísticamente significativos entre 1992 y 1998, excepto en las áreas rurales en 1994, controlado por nivel de ingreso, lugar de residencia, género, educación y edad del jefe del hogar y por el tamaño del hogar.

\section{Discusión}

La prevalencia ajustada de los hogares que reportaron algún gasto en tabaco disminuyó de 22.4 a $9.9 \%$ entre 1992 y 1998. Los hogares asignaron poco más de $4 \%$ de su ingreso a la adquisición de tabaco. Se observó un gradiente entre nivel de ingreso y gasto en cigarrillos. El quintil I (el más pobre) destinó una mayor proporción de su ingreso que los quintiles superiores. El promedio de cigarrillos consumidos diariamente pasó de 7.5 a 9.8 entre 1992 y 1998. Se estimó que más de $90 \%$ de los "hogares fumadores" consumió hasta una cajetilla diaria. La demanda estimada de cigarrillos sin filtro aumentó entre 1992 a 1998, pasando de 0.4 a 4.8\%, registrándose el mayor incremento en 1996. Finalmente, se encontró que, tanto los precios como el ingreso, fueron los determinantes más importantes del gasto en tabaco.

Como se mencionó, la demanda de tabaco se manejó bajo dos niveles de análisis: el gasto y el consumo. La ENIGH no consigna información sobre el número de personas que consumen tabaco al interior de cada vivienda encuestada ni capta la posibilidad de "adicciones" entre los hogares, sino el nivel de gasto y consumo hecho por las familias en un satisfactor que entra en su canasta de consumo, por lo que, no puede distinguir que no todo el gasto en tabaco implica el consumo de cigarrillos y a la inversa, todo el consumo de éstos no necesariamente implica un gasto. Sin embargo, la ENIGH asocia con cada consumo un gasto, por lo tanto, nuestra variable de aproximación de la demanda fue el consumo semanal de cajetillas. Las variables socioeconómicas de control fueron precio, ingreso semanal, edad, género, tamaño del hogar y educación. La estratificación en quintiles de ingreso trimestral real tiene el inconveniente de que a nivel intraquintil, la diferencia de ingresos de los hogares es aún muy grande, por lo que los resultados obtenidos podrían desviarse de los hallazgos reportados por la literatura.
La relación del ingreso y la edad del jefe del hogar con la demanda es directa y estadísticamente significativa, es decir, ante incrementos en estas variables, el consumo de tabaco también aumenta. El coeficiente positivo (b) del ingreso fortalece la propuesta de precios como mecanismo para reducir la demanda, ya que si éste se incrementa y el ingreso se mantiene constante, la capacidad adquisitiva disminuye y, por ende, el consumo. Es conocido que el consumo se incrementa con la edad, por ejemplo en la ENA de 1998 se encontró que el promedio de cajetillas consumidas anualmente entre jóvenes 15 a 24 años fue de 164.3 mientras que entre los adultos de 50 y 65 años fue de 326.5 cajetillas. Este resultado es coherente con la llamada "Teoría Económica de la Adicción", que sostiene que el consumo pasado afecta positivamente el consumo presente. ${ }^{15}$

La asociación inversa entre precios y consumo de tabaco antes descrita, apoya la hipótesis de que una política de precios, basada en impuestos a los cigarrillos, puede impactar positivamente en una reducción de la demanda. Una de las maneras que puede ser efectiva para bloquear el acceso a los cigarros de los adolescentes y jóvenes sería aplicar mayores impuestos a los productos que contienen tabaco y así desalentar el consumo. Por su edad, generalmente aún no son adictos y se conoce que son un grupo muy sensible a los cambios en el precio, sobre todo en los países de bajos y medianos ingresos. ${ }^{27} \mathrm{Se}$ ha informado que para menores de 25 años la elasticidad del precio del cigarro es mayor que entre personas más adultas, lo que quiere decir que frente al alza de los precios muchos adolescentes y jóvenes abandonan el hábito ante la imposibilidad de poder pagarlo. Lo anterior, unido a que es poco probable que quien haya llegado a los 25 años sin fumar comience a hacerlo, justifica los impuestos al tabaco como medida para frenar el consumo entre adolescentes y jóvenes. ${ }^{28}$

La demanda de tabaco en México para el periodo 1992-1998 ha mostrado cambios significativos en casi todos sus rubros. La demanda se describió bajo dos niveles de análisis: el gasto observado, tanto en prevalencia de hogares como en el porcentaje del ingreso destinado al mismo, y el consumo estimado por cajetillas y cigarrillos, así como en la estimación de la prevalencia de hogares demandantes de cigarrillos sin filtro o "baratos".

A pesar de que la prevalencia de hogares que reportaron algún gasto en tabaco disminuyó significativamente de 22.4 a $9.9 \%$ entre 1992 y 1998, los cambios observados en la fracción del ingreso destinado al consumo de tabaco no fueron estadísticamente significa- 
tivos, pero sí lo fueron las variaciones en el consumo diario de cigarrillos y en la prevalencia de hogares que consumen más de una cajetilla diaria. Esto quiere decir que los hogares fumadores aumentaron su demanda de tabaco sin afectar el gasto destinado a éste, situación posiblemente explicada por consumo de marcas más baratas o compra ilegal del producto.

La reducción en el número de "hogares fumadores" y el aumento en el consumo diario de cigarrillos, así como el incremento en la prevalencia de hogares consumidores de más de una cajetilla diaria sugiere que los "hogares fumadores" leves han empezado a desaparecer de las ENIGH y, por consiguiente, éstas sólo captan a los grandes "hogares fumadores". Cabe destacar que la ENIGH reporta el gasto declarado por los adultos (jefe del hogar) y no por los jóvenes del hogar que pueden consumir tabaco, gasto que no es detectado por la ENIGH. Igualmente, esta encuesta no registra el gasto en tabaco declarado por los hogares, realizado antes de la semana de la encuesta.

La estimación de la demanda de cigarrillos sin filtro o "baratos" mostró una tendencia creciente entre 1992 y 1996, pasando de 0.4 a 11.4\%, respectivamente. Para 1998, esta cifra descendió menos de siete puntos porcentuales respecto de 1996, ubicándose este indicador en $4.8 \%$. Estas estimaciones coinciden con la información obtenida de la Encuesta Industrial Anual, que reporta un crecimiento de la producción de cigarrillos sin filtro entre 1994 y 1996 de más de 330 millones, mientras que entre 1996 y 1998 dicha producción decreció en poco más de 285 millones de cajetillas (figura 1.3). Los "hogares fumadores" pobres (quintil I) fueron mayores demandantes de este tipo de cigarrillo que los "hogares fumadores" ricos (quintil V). Una explicación de esta traslación de la demanda de cigarrillos con filtro a sin filtro, entre 1992 y 1996, es que la crisis económica de finales de 1994 provocó la pérdida en la capacidad adquisitiva de la población, lo que motivó que los hogares buscaran alternativas de consumo de tabaco que no afectaran considerablemente su nivel de ingreso.

Se puede hipotetizar, por un lado, que los fumadores activos cuando ven deteriorada su capacidad adquisitiva buscan diversificar su demanda, consumiendo cigarrillos "baratos" que no lesionan severamente su economía. Aunque también recurren a los mercados informales de cigarrillos. Por lo anterior se plantea que los impuestos al tabaco son distorsionantes, lo que genera temor en los gobiernos de ver reducidos sus ingresos fiscales. Este resultado tiene consecuencias severas para los estratos de ingreso bajo, ya que de acuerdo con la Encuesta de Fumadores del
Estado de Morelos se encontró que los niveles medios de cotinina en saliva eran mayores en fumadores de cigarrillo sin filtro que en aquellos que fumaban cigarrillo con filtro. ${ }^{10}$ Con este resultado puede decirse que este tipo de tabaco representa un subsidio a la enfermedad de los hogares con recursos escasos.

El incremento en el número de cigarrillos fumados diariamente (dos cigarrillos) y particularmente la situación de que en 1998 el consumo de los quintiles I y V halla sido el mismo, tiene implicaciones severas en términos de salud porque los residentes del quintil más pobre no tienen acceso financiero a la atención y mucho menos a terapias de remplazo.

Los resultados del modelo de regresión de la demanda permitieron observar que los precios y el ingreso, así como la edad, fueron los factores más importantes para explicar el consumo de tabaco. Estos sugieren que una política de precios podría favorecer la reducción de los "hogares fumadores" y, por ende, el consumo de cigarrillos (figura 1.4). Asimismo, la elaboración de planes y programas anti-adicciones enfocados a la población infantil podrían mejorar las expectativas de salud de la población.

En México, los impuestos a los cigarrillos pueden ser considerados como uno de los mecanismos más importantes, no solamente para disminuir el consumo de tabaco sino para generar recursos fiscales que permitan el financiamiento de la atención a la salud de los fumadores. Para ello, resulta de primera necesidad estimar los costos asociados con la atención de las enfermedades atribuibles al tabaquismo, la elasticidad del precio de la demanda de tabaco, así como la fracción de ingresos fiscales atribuibles al consumo de cigarrillos.

Sin embargo, se debe tener en consideración que la política fiscal del tabaco puede ocasionar efectos negativos en el bienestar económico de los "hogares fumadores" pobres, ya que como se observó, los quintiles pobres destinan una proporción importante de su ingreso a satisfacer su adicción (10\%), es decir, los impuestos son regresivos ya que recaen en los estratos de ingresos bajos. Resultaría interesante hacer estudios de evaluación económica que comparen los gastos futuros ocasionados por la atención de las patologías atribuidas a tabaco de la población fumadora pobre, sin seguridad social, con un impuesto regresivo que los motive a cesar el consumo.

A manera de recomendación sugerimos que en futuras aplicaciones de la ENIGH, se recoja la marca del cigarrillo consumido, así como su precio. Adicionalmente, la información sobre el número de fumadores por hogar y los miembros que tienen el hábito. Esta 
información sería de valiosa contribución para mejorar la precisión en los estudios, tanto epidemiológicos como económicos.

\section{Referencias}

1. Lazcano EC, Tovar V, Meneses F, Rascón RA, Hernández M.Trends of lung cancer mortality in México. Med Res 1997;28(4):565.

2. Kuri P, Jesús M, Tapia R. Riesgos para la salud y beneficios del abandono. México, D.F.: Secretaría de Salud, 1998:13-16.

3. Barendregt JJ, Bonneux L,Van Der Maas PJ. The health care costs of smoking. N Engl J Med 1997;337:1052-1057.

4.Luce BR, Schweitzer SO. Smoking and alcohol abuse:A comparison of their economic consequences. N Engl J Med 1978;298(10):422-435.

5. Miller VP, Ernst C, Collin F. Smoking-atributable medical costs in USA. Soc Sci Med 1999;48: .

6. N ewsweek 2000;5(32):45-49.

7.Tobacco C ontrol Profiles. $11^{\text {th }}$ W orld C onference on Tobacco 0 R Health 2000. Atlanta (GA):A merican Cancer Society, 2000.

8. Tapia R, Lazcano F. El consumo de tabaco en México. Resultado de la Encuesta $N$ acional de Salud. Bol Epidemiol Mex 1989;4:33.

9.Tapia R, Lazcano F, Medina ME. El hábito de fumar en la Ciudad de México. Boletín de Epidemiología Mex 1990;5:37.

10. C ampuzano JC, Salmerón J, Lazcano E, C evazo M, Kuri P, Samet J et al. Cotinine in sample of Mexican's smokers. In process of publication.

11. Instituto $N$ acional de Estadística, Geografía e Informática. Encuesta Industrial A nual. A guascalientes:IN EGI, 1992, 1994, 1996 y 1998.

12. Instituto N acional de Estadística, Geografía e Informática. Sistema de Cuentas N acionales de México, Cuentas de Bienes y Servicios 1988-1999, Tomo I.A guascalientes: IN EGI, 2000.

13. Chalo upka F, Prabhat J, ed. Tobacco Control in D eveloping Countries. N uevaYork (N Y): 0 xford University Press, 2000.
14. Chaloupka F, Grossman M. Price, tobacco control policies and youth smoking. Cambridge (MA): N ational Bureau of Economic Research, 1996; W orking paper N 0.5740.

15. Chaloupka F,W arner K.The economics of tobacco. $N$ ational Bureau of Economic Research, 1999; Working paper N 0. 7047.

16. Flewelling R, Kenney $E$ et al. First-year impact of the 1989 California cigarette tax increase on cigarette consumption. Am J Public Health 1992;82:867-869.

17. Keeler $\mathrm{E}, \mathrm{Hu} W$, et al. Taxation, regulation and addiction: A demand function for cigarettes based on times series evidence. J Health Econ 1993;12:1-18.

18. Meier K, Licari M.The effect of cigarette taxes on cigarette consumption, 1955 through 1994. Am J Public Health 1997;87:1126-1130.

19. Becker G, Murphy K. A theory of rational addiction. J Pol Econ 1988:96:675-700.

20. Becker G, Murphy K.A simple theory of advertising as a good or bad. Q uarterly J Econ 1993;941-964.

21. Becker G, Grossman M, Murphy K. An empirical analysis of cigarette addiction. Am Econ Rev 1994;84:396-418.

22. Galbraith J, Kaisserman M. Taxation, smuggling and demand for cigarettes in Canada: Evidence from times-series data. J Health Econ 1997;16:287-301.

23. Moore M. D eath and tobacco taxes. C ambridge (MA): N ational Bureau of Economic Research, 1996; W orking paper N 0.5153.

24. Suranovic S, Goldfarb R, Leonard T. An economic theory of cigarette addiction.J Health Econ 1997;18:1-29.

25. Instituto Nacional de Estadística, Geografía e Informática. Encuesta $\mathrm{N}$ acional de Ingreso y $\mathrm{G}$ asto de los Hogares. México.A guascalientes: IN EG I, 1992, 1994, 1996 y 1998.

26. Instituto Nacional de Estadística, Geografía e Informática. Encuesta $\mathrm{N}$ acional de Ingreso y Gasto de los Hogares. Manual del Entrevistador. México, A guascalientes: IN EG I, (1992, 1994, 1996 y 1998).

27. O rganización Panamericana de la Salud, Banco Mundial. La epidemia del tabaquismo. Los gobiernos y los aspectos económicos del control del tabaco.W ashington, DC: 0 PS, 2000;Publicación científica N 0.577.

28. Lewit EM.:The effects of government regulation on teenage smoking.J Law Econ 1981;24 december:545-575. 